\title{
Penile prosthesis implantation and tunica albuginea incision without grafting in the treatment of Peyronie's disease with erectile dysfunction
}

\begin{abstract}
Miroslav L Djordjevic and Vladimir Kojovic
We evaluated penile prosthesis implantation with tunica albuginea-relaxing incisions without grafting in the treatment of Peyronie's disease associated with erectile dysfunction. Between April 2005 and June 2011, 62 patients underwent surgery due to severe Peyronie's disease associated with erectile dysfunction. Malleable and inflatable penile prostheses were inserted in 49 and 13 cases, respectively. Penile prostheses were inserted into the corpora cavernosa using the standard ventral approach. After lifting the neurovascular bundle, the tunica albuginea was incised and opened at the plaque region to correct the deformities and to lengthen the penis. Subsequently, the wide neurovascular bundle was replaced, and all incisions of the tunica albuginea were covered to prevent corporal grafting. In the median follow-up of 35 months (range 14-82 months), the penis was completely straightened in 59 (95\%) patients. Numbness of the glans, which the patients found initially upsetting, decreased or disappeared spontaneously 3-6 months later. Penile prosthesis implantation with tunica albuginea incisions is a viable alternative in the treatment of Peyronie's disease because the extensive dissection of the neurovascular bundle allows a good approach to the plaque and provides excellent covering of the incised tunica albuginea without additional grafting.
\end{abstract} Asian Journal of Andrology (2013) 15, 391-394; doi:10.1038/aja.2012.149; published online 25 February 2013

Keywords: erectile dysfunction; neurovascular bundle; penile prosthesis; Peyronie's disease; tunica albuginea

\section{INTRODUCTION}

Peyronie's disease is an acquired fibrotic disease of the tunica albuginea, characterized by a fibrotic plaque that causes penile deformities, shortening, loss of penile flexibility and associated sexual dysfunction. ${ }^{1}$ The conservative management of Peyronie's disease has been shown to be of limited efficacy in many previous reports, and it is usually reserved for mild forms of the disease. As a result, surgical approaches remain the gold standard for treatment of Peyronie's disease but should be considered after disease stabilisation. ${ }^{2}$ Several procedures were described to correct the curvature. Some of the well-known and frequently employed surgical techniques include tunical shortening procedures such as tunical plication. Penile lengthening procedures are reserved for patients with preserved erectile function and severe penile length loss followed by marked hourglass deformities. When erectile dysfunction is associated with Peyronie's disease, penile prosthesis implantation with or without plaque surgery is the standard of care.

In the last decade, modelling the penis over a penile prosthesis has been reported and has become very popular, with an $86 \%$ success rate. ${ }^{3}$ However, other authors have reported significant residual curvature in $20 \%-30 \%$ of patients after simple modelling that required additional plaque surgery to achieve a straightened penis. ${ }^{4}$ Reconfiguration of the tunica albuginea with penile straightening can be performed by tunical incision with or without additional grafting of the tunical defect. ${ }^{5,6}$ We evaluated our experience in 62 patients with Peyronie's disease and erectile dysfunction in whom a penile prosthesis was implanted; deformities were corrected using simple incisions of the affected tunica albuginea and a well-mobilized neurovascular bundle was used to cover defects without grafting.

\section{MATERIALS AND METHODS}

Between April 2005 and June 2011, 62 patients (aged 55-69 years) with severe Peyronie's disease and erectile dysfunction underwent penile prosthesis implantation, tunica albuginea incisions and reconfiguration of the deformed penis without grafting of the tunical defects. The preoperative evaluation included a medical and sexual history with the international index of erectile function (IIEF) questionnaire, a physical examination and colour Doppler sonography during a pharmacological erection induced by an intracavernosal injection of prostaglandin E1. The indication for penile prosthesis implantation was based on a significant reduction of penile rigidity caused by severe penile deformities or vasculogenic impairment. The median preoperative duration of Peyronie's disease was 31 months (range 12-73 months). A severe decrease of penile length due to Peyronie's disease was reported in 53 of $62(85 \%)$ patients. Surgery was performed when the plaque was mature and the curvature had been stable for at least 9 months. Patients with ventral penile curvature and calcified plaques were excluded from the study and treated with grafting of the tunical defects. Malleable and inflatable penile prostheses (AMS 700 series; American Medical System, Minnetonka, MN, USA) were inserted in 49 and 13 cases, respectively. None of the patients in our series had undergone previous penile surgery. Incising the tunica albuginea resulted in defects ranging from 14 to $28 \mathrm{~mm}$ (median $19 \mathrm{~mm}$ ) in diameter. 


\section{Operative technique}

The penis was typically degloved using a subcoronal circumcision incision. The skin and underlying tissue were dissected along the length of Buck's fascia layer to the root of the penis. An artificial erection was induced to reveal the degree of the deformity, which ranged from $45^{\circ}$ to $85^{\circ}$. The neurovascular bundle was completely elevated from the tunica albuginea starting from the coronal level and reaching the base of the penis (Figure 1). The dissection continued very close to the tunica albuginea towards the base of the penis; it was lifted with the Buck's fascia to provide easier dissection of the neurovascular bundle, while Buck's fascia makes the neurovascular bundle thicker, wider and stronger (Figure 2). Laterally, the dissection was performed close to the urethra to the Buck's fascia with the aim of creating a wide neurovascular bundle to cover the tunical defects after correction of the penile deformities. If the plaque was incorporated into the neurovascular bundle, the latter was dissected through the plaque. A portion of the plaque remained attached to the neurovascular bundle, while its other portion remained within the tunica albuginea. This procedure completely preserved all structures of the neurovascular bundle and thinned the plaque within the tunica albuginea. The ventral surface of the tunica albuginea was approached, and two conventional longitudinal corporotomies were created. Under direct vision, the corpora cavernosa were dilated up to $14 \mathrm{~mm}$ using Hegar dilators. Care was taken to dilate the corpora close to the ventral tunica albuginea to prevent disruption of the dorsal side. The length of the corpora was precisely measured in a maximal stretched position with a Furlow inserter. The length of the penile prosthesis was defined according to the length of the ventral surface of the corpora cavernosa. Penile prostheses were inserted, and residual curvature was assessed. One or more relaxing transverse incisions ( $\mathrm{H}$ shape) were made using cautery at the plaque region from one side to the other to correct the deformities and to lengthen the penis. Incisions were made carefully over the prostheses and were limited to the tunica, preserving the underlying cavernosal tissue and preventing damage of the inflatable cylinders (Figure 3). Before closure of the corporotomies, prosthesis adequacy and length were evaluated. In 11 cases (17.74\%) with inappropriate prosthesis length, the prosthesis was removed and replaced with the correct size. The corporotomies were closed with 2-0 polydioxanone running sutures. The thick, strong and wide neurovascular bundle was replaced over the dorsal side of the penis covering the incision sites, and, thus, grafting of the tunica albuginea was avoided (Figure 4). In cases with an inflatable penile prosthesis, a pump was inserted into a superficial scrotal pocket through a separate incision,

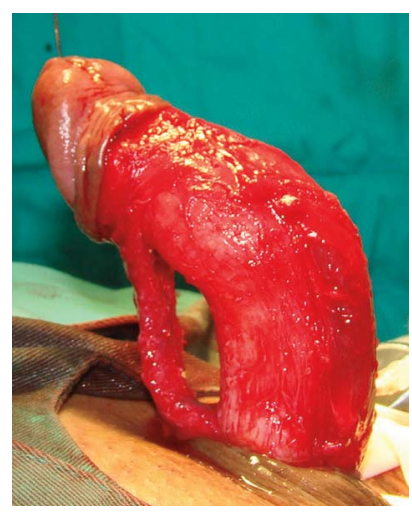

Figure 1 The neurovascular bundle is lifted from the corpora cavernosa. Severe dorsal curvature is visible due to artificial erection. and the reservoir was placed in the typical suprapubic location. After completing the connections and verifying correct implant function, the penile body skin was reconstructed, including a circumcision to prevent phimosis or other preputial deformities (Figure 5). The inflatable prosthesis was left in the partially inflated condition during initial healing to reduce cicatrix contracture and recurrent curvature. The penis was dressed with an elastic bandage, and a Foley catheter was inserted for 1 day. Prophylactic antibiotics were used in all patients for 7 days.

\section{RESULTS}

The median follow-up was 35 months (range 14-82 months). The penis was completely straightened in 59 (95\%) patients. Penile rigidity was improved in all cases. The overall mean increase in penile length was $2.1 \mathrm{~cm}$ (range $1.7-4.1 \mathrm{~cm}$ ) in comparison to the preoperative length. Three patients developed a mild residual curvature $\left(<20^{\circ}\right)$ during the follow-up period, but this had no impact on sexual intercourse. During the follow-up period, there were no infections or mechanical failure of the prostheses.

A decrease in penile girth was reported in seven of 49 patients with a malleable penile implant, and in three patients, it was replaced with an inflatable prosthesis 1 year later. Hypermobility of the glans occurred in five patients due to the plaque location under the glans cap and its partial lifting, requiring surgical repair. The glans cap was dissected ventrally and fixed to the corpora cavernosa in the proper position. Numbness of the glans, which the patients found initially upsetting, was noted in 23 cases, but numbness decreased and disappeared spontaneously within 6 months. Glans necrosis did not occur in our series.

The IIEF questionnaire was used to obtain sexual life and satisfaction data (Table 1). According to the results, all domains were improved significantly in the postoperative questionnaire compared to the preoperative questionnaire $(P<0.05 ; t$-test $)$. Analysing the surgical outcome, 58 patients ( $94 \%$ ) were very satisfied and did not regret their decision to undergo this type of surgery.

\section{DISCUSSION}

The management of Peyronie's disease creates a dilemma in the choice of treatment and potential improvement. Many surgical procedures are used to repair penile deformities, and the choice is dependent on the type of deformity, penile length and surgeon's preference. The criteria for the plication technique are good potency and adequate penile length. In patients with a shortened penis, marked curvature or an hourglass deformity, lengthening procedures with grafting offer the best possible gain from a reconstruction standpoint. ${ }^{7}$ Penile prosthesis

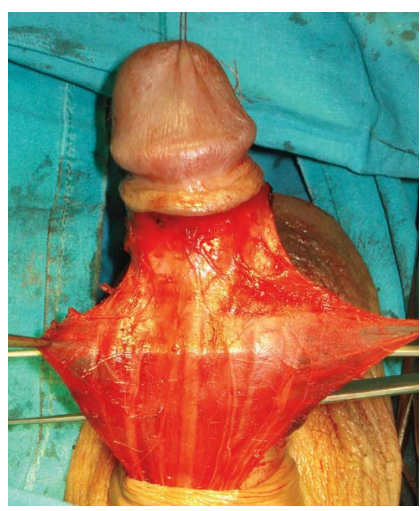

Figure 2 The appearance of a completely dissected neurovascular bundle. All elements are preserved. 


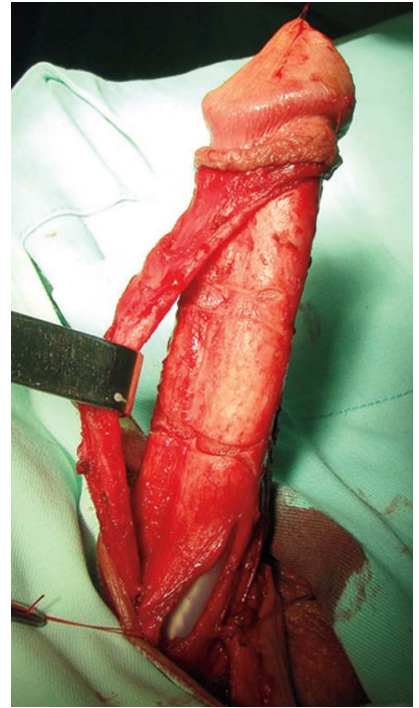

Figure 3 Two relaxing incisions are made at the plaque region. Malleable prostheses are inserted through the ventral longitudinal corporotomies.

implantation is the standard procedure for patients with Peyronie's disease and concomitant erectile dysfunction that does not respond to medical treatment. ${ }^{1,8}$ The standard algorithm for these patients includes implantation of a penile prosthesis, incision of the plaque and grafting of the defect. In patients with mild degrees of curvature, straightening can be achieved with prosthesis implantation alone. ${ }^{9}$

Manual modelling over the penile prostheses was first described by Wilson and Delk ${ }^{3}$, who reported successful straightening in $86 \%$ of patients; however, $8 \%$ of men required subsequent plaque surgery, and urethral laceration occurred in $4 \%$ of patients. Penile prosthesis implantation combined with modelling can provide penile straightening in more than half of patients, but up to $20 \%-30 \%$ of patients require additional surgery (incisions with or without grafting). ${ }^{10}$ Montorsi et al. ${ }^{11}$ introduced the concept of multiple relaxing incisions of the tunica albuginea followed by immediate implantation of a penile prosthesis to achieve complete penile straightening, maximal lengthening and adequate penile rigidity. Both malleable and inflatable implants are available, but inflatable prostheses are associated with higher functional satisfaction and lower persistent curvature rates. In their study, Montorsi et al. ${ }^{11}$ reported satisfaction in only $48 \%$ of the patients who underwent semirigid penile prosthesis implantation. However, there are several studies with inflatable prosthesis implantation in which the success rates were $84 \%-100 \% .^{6,9,12,13}$ Transcorporal plaque incision presents a novel minimally invasive endoscopic approach with high success rates for penile straightening and lengthening.

Based on previous studies and our experience in neurovascular bundle dissection for the curvature correction of severe congenital and acquired penile anomalies, we started to use tunica albuginea incisions followed by penile prosthesis implantation without grafting in the treatment of Peyronie's disease with associated erectile dysfunction. ${ }^{14,15}$ An approach to the plaque to correct curvature and other penile deformities is difficult in Peyronie's disease, particularly if the deformities are in the distal third of the corpora cavernosa. The neurovascular bundle, which is often incorporated into the plaque, particularly if calcified, can be easily injured using standard techniques (including the midline approach to the plaque region) during its lifting. We started by lifting the neurovascular bundle with the Buck's fascia ventrally from the urethra to the dorsal side of the penis. This

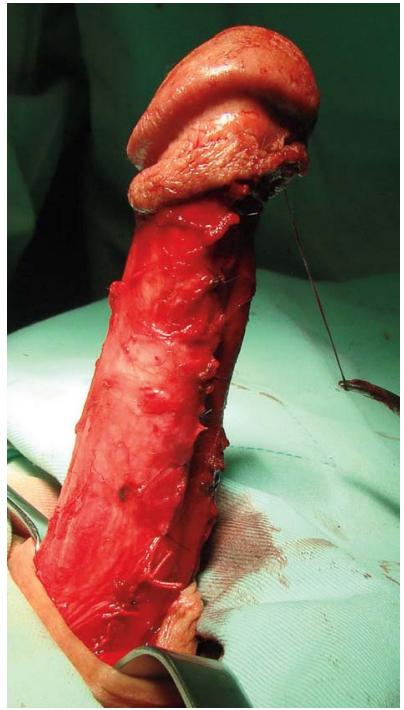

Figure 4 The wide neurovascular bundle completely covers all tunical defects, and, thus, grafting of the tunica is not necessary.

provides easier dissection, while the Buck's fascia makes the bundle thicker, wider and stronger. This procedure completely preserves all structures of the neurovascular bundle and allows dissection through the plaque so that a portion of the plaque remains attached to the neurovascular bundle, while the other part remains within the tunica albuginea. This enables thinning of the plaque within the tunica albuginea and decreases the degree of penile deformity; the corporal bodies become completely free and mobile. This approach to the plaque region allows the excellent repair of all penile deformities. One or more relaxing incisions enable complete straightening and additional lengthening of the affected corporeal bodies. Finally, a completely preserved, thick, strong and very wide neurovascular bundle covers all incision sites, avoiding tunica albuginea grafting.

However, there are disadvantages to the procedure as well, including temporarily decreased glans sensitivity and glans mobility requiring surgical revision. In the majority of our patients (79\%), malleable prostheses were used (because their medical insurance did not cover the additional expense), resulting in decreased penile girth in some patients and the need for replacement with inflatable prostheses. Additionally, the prosthesis measurement before plaque incision is not precise and could be problematic because the length of the corpora cavernosa changes greatly before and after the relaxing incisions. In 11 cases, the removal of the prosthesis and replacement with the correct length was simple and safe.

However, there is a limitation to the use of both groups of patients in any comparative study due to wide differences between the types of prosthesis and the different numbers of patients. The lack of standardized methods for recording long-term complications and sexual function outcomes makes the direct comparison of our results limited. Therefore, although a consensus on the ideal method for the repair of penile deformities may never be reached, current efforts should focus on reaching a consensus on the ideal way to follow these patients in the long term.

Our initial concerns about this procedure included the possibility of haematoma, bulging or herniation of the cylinders through the incision defects, but none of these occurred during the follow-up period. This technique is limited in cases with severe degrees of curvature and calcified plaques, for which grafting of the incised tunica albuginea is recommended. ${ }^{13,16,17}$ 


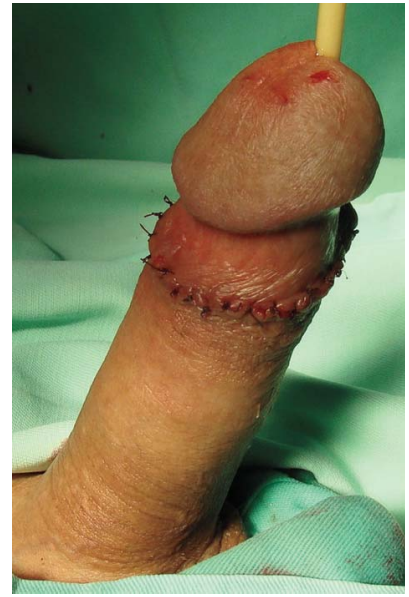

Figure 5 Final appearance of the penis is completely straightened.

In addition to other techniques, we believe that the use of extended incisions of the tunica albuginea provides an additional viable alternative within our operative armamentarium in the management of this severe problem. Our experience suggests that this technique leads to the satisfactory straightening of the penis, a low rate of curvature recurrence/ persistence and an absence of urethral injury. Complete dissection of the neurovascular bundle offers better visualisation of the plaque region, a direct approach for incisions of the tunica albuginea and complete control of the implanted prostheses. Lastly, a wide and strong neurovascular bundle completely covers all corporeal defects, avoiding additional grafting and time-consuming procedures. This technique is limited in cases with severe degrees of curvature and calcified plaques, for which grafting of the incised tunica albuginea is recommended.

\section{AUTHOR CONTRIBUTIONS}

MLD designed and coordinated the study, performed all surgical procedures, observed and interviewed the patients during follow up, wrote and revised the manuscript. VK participated in surgical procedures, followed the patients, collected the questionnaire files, analyzed the output data, helped to write and draft the manuscript.

\section{COMPETING FINANCIAL INTERESTS}

The authors declare that they have no competing financial interests.
Table 1 The mean preoperative and postoperative scores of various IIEF domains $(\boldsymbol{P}<0.05, t$-test $)$

\begin{tabular}{lccc}
\hline Domain & Preoperative scores & Postoperative scores & $P$ \\
\hline Erectile function & 8.2 & 28.2 & $<0.001$ \\
Orgasmic function & 5.1 & 7.9 & $<0.001$ \\
Sexual desire & 7.2 & 8.2 & $<0.01$ \\
Intercourse satisfaction & 2.9 & 10.2 & $<0.001$ \\
Overall satisfaction & 2.8 & 8.9 & $<0.001$ \\
\hline
\end{tabular}

Abbreviation: IIEF, international index of erectile function.

\section{ACKNOWLEDGMENTS}

This study was supported by the Ministry of Science, Republic of Serbia, Project No. 175048 .

1 Ralph D, Gonzalez-Cadavid N, Mirone V, Perovic S, Sohn M et al. The management of Peyronie's disease: evidence-based 2010 guidelines. J Sex Med 2010; 7: 2359-74.

2 Kadioglu A, Kucukdurmaz F, Sanli O. Current status of the surgical management of Peyronie's disease. Nat Rev Urol 2011; 8: 95-106.

3 Wilson SK, Delk JR. A new treatment for Peyronie's disease: modeling the penis over an inflatable penile prosthesis. J Urol 1994; 152: 1121-3.

4 Mulcahy JJ, Wilson SK. Management of Peyronie's disease with penile prostheses. Int J Impot Res 2002; 14: 384-8.

5 Shaeer O. Trans-corporal incision of Peyronie's plaques. J Sex Med 2011; 8: 589-93.

6 Montorsi F, Salonia A, Maga T, Colombo R, Cestari A et al. Reconfiguration of the severely fibrotic penis with a penile implant. J Urol 2001; 166: 1782-6.

7 Egydio PH. Surgical treatment of Peyronie's disease: choosing the best approach to improve patient satisfaction. Asian J Androl 2008; 10: 158-66.

8 Smith JF, Walsh TJ, Lue TF. Peyronie's disease: a critical appraisal of current diagnosis and treatment. Int J Impot Res 2008; 20: 445-9.

9 Chaudhary M, Sheikh N, Asterling S, Ahmad I, Greene D. Peyronie's disease with erectile dysfunction: penile modeling over inflatable penile prostheses. Urology 2005; 65: 760-4.

10 Kadioglu A, Akman T, Sanli O, Gurkan L, Cakan M et al. Surgical treatment of Peyronie's disease: a single center experience with 145 patients. Eur Urol 2008; 53: 432-9.

11 Montorsi F, Guazzoni G, Bergamaschi F, Rigatti P. Patient-partner satisfaction with semirigid penile prostheses for Peyronie's disease: a 5-years follow-up study. J Urol 1993; 150: 1819-21.

12 Carson CC. Penile prosthesis implantation in the treatment of Peyronie's disease and erectile dysfunction. Int J Impot Res 2000; 12: 122-6.

13 Levine LA, Benson J, Hoover C. Inflatable penile prosthesis placement in men with Peyronie's disease and drug-resistant erectile dysfunction: a single-center study. J Sex Med 2010; 7: 3775-83.

14 Perovic SV, Djordjevic ML, Djakovic N. A new approach to the treatment of penile curvature. J Urol 1998; 160: 1123-7.

15 Perovic SV, Djordjevic ML. Penile disassembly technique in the surgical treatment of Peyronie's disease. Br J Urol 2001; 88: 731-8.

16 Djinovic R. Penile corporoplasty in Peyronie's disease: which technique, which graft? Curr Opin Urol 2011; 21: 470-7.

17 Serefoglu EC, Hellstrom WJ. Treatment of Peyronie's disease: 2012 update. Curr Urol Rep 2011; 12: 444-52. 